\title{
Trends in Vitamin C Consumption in the United States: 1999-2018
}

\author{
Mary Brauchla, Mark J. Dekker and Colin D. Rehm * (C)
}

PepsiCo, 700 Anderson Hill Road, Purchase, NY 10577, USA; Mary.Brauchla@pepsico.com (M.B.);

Mark.Dekker@pepsico.com (M.J.D.)

* Correspondence: Colin.Rehm@pepsico.com; Tel.: +1-(914)-253-2432

check for updates

Citation: Brauchla, M.; Dekker, M.J; Rehm, C.D. Trends in Vitamin C Consumption in the United States: 1999-2018. Nutrients 2021, 13, 420. https://doi.org/10.3390/nu13020420

Academic Editor: Anitra Carr

Received: 11 January 2021

Accepted: 27 January 2021

Published: 28 January 2021

Publisher's Note: MDPI stays neutral with regard to jurisdictional claims in published maps and institutional affiliations.

Copyright: (c) 2021 by the authors. Licensee MDPI, Basel, Switzerland. This article is an open access article distributed under the terms and conditions of the Creative Commons Attribution (CC BY) license (https:// creativecommons.org/licenses/by/ $4.0 /)$.

\begin{abstract}
Low intakes of fruits and vegetables have resulted in suboptimal intakes of several micronutrients, including vitamin C. This cross-sectional study used data from 84,902 children/adults ( $\geq 1$ y) who completed a 24-h dietary recall as part of the United States National Health and Nutrition Examination Survey (1999-2018). Mean vitamin C intakes from foods/beverages were calculated as were trends in major food/beverage sources of vitamin C. Percentages below the Estimated Average Requirement (EAR) were estimated. Overall, mean vitamin C consumption declined by $23 \%(97-75 \mathrm{mg} / \mathrm{d}$; $p$-value for trend < 0.001). 100\% fruit juice was the leading source of vitamin C ( $25.6 \%$ of total or $21.7 \mathrm{mg} / \mathrm{d})$, but this declined by $48 \%(25-13 \mathrm{mg} / \mathrm{d}$; $p$-value for trend $<0.001)$. Whole fruit increased among children/adolescents $(+75.8 \% ; 10-17 \mathrm{mg} / \mathrm{d} ; p$-value for trend $<0.001)$, but not adults, while the vegetable contribution was generally unchanged. The proportion of the population below the EAR increased by $23.8 \%$ on a relative scale or 9 percentage points on an absolute scale (38.3-47.4\%). Declines in vitamin C intake is driven largely by decreases in fruit juice coupled with modest increases in whole fruit. Due to associations between vitamin $C$ intake and numerous health outcomes these trends warrant careful monitoring moving forward.
\end{abstract}

Keywords: vitamin C; dietary surveys; trends; descriptive studies; United States; fruit juice; fruit; vegetables

\section{Introduction}

Most of the United States (US) population currently fails to meet fruit and vegetables recommendations provided by the most recent Dietary Guidelines for Americans (DGAs) [1]. Recent data from the National Health and Nutrition Examination Survey (NHANES) suggests the average fruit and vegetable intake in US adults is 0.9 cup equivalents (cup eq.) and 1.5 cup eq., respectively, which is far below the recommended 1.5-2 cup eq. of fruits and $2-3$ cup eq. of vegetables [2,3]. Low consumption of fruits and vegetables have led to low intake of several vitamins and minerals, including vitamin $C[1,4]$. Many fruits and vegetables contain high concentrations of vitamin $C$ and commonly promoted sources in the US include red and green bell peppers, citrus fruit and juices, strawberries and kiwi [5]. Vitamin $C$ is also added to foods and beverages to improve the nutrient profile or for other purposes, including flavoring and food preservation.

The health benefits of vitamin $C$ are well documented: vitamin $C$ is essential to normal function of the immune system [6-8], skin health and collagen synthesis [9], and function of the nervous system [10]. Meta-analyses of epidemiologic studies have shown that vitamin $\mathrm{C}$ measured via self-report and in sera is associated with a decreased risk of coronary heart disease, stroke and some cancers, as well as all-cause mortality [11] although whether these associations are directly related to vitamin $C$ itself or driven by vitamin $C$ as a marker of fruit and vegetable intake is less certain [12]. In order to ensure normal function of the body and to avoid vitamin $\mathrm{C}$ deficiency current US vitamin $\mathrm{C}$ recommendations are to consume $75 \mathrm{mg}$ /day for women and $90 \mathrm{mg} /$ day for men [13]. Severe vitamin C deficiency is rare but 
if vitamin C consumption is limited or nonexistent for several weeks it can result in scurvy, a disease causing anorexia, poor wound healing, and tooth loss and gingival bleeding [14].

Despite the clear benefits of adequate vitamin $C$ intake this nutrient has consistently been classified as underconsumed in the DGAs since $2005[1,15,16]$, though not as a nutrient of public health concern. Surprisingly, there is little data documenting the most commonly consumed sources of vitamin $C$ and how changes in intake of top sources affect intakes. A better understanding of vitamin $C$ consumption patterns and trends could provide valuable information to promote intake in the future and monitor for any upticks in potential sub-optimal intakes. The purpose of this study was to track vitamin $C$ intake, percentage of the population consuming amounts below the Estimated Average Requirement (EAR) and to identify top food and beverage sources of vitamin $C$ in a large nationally-representative sample of the US population over a 20 -year period.

\section{Materials and Methods}

\subsection{Data Sources}

This cross-sectional study was based on data from the 10 most recent 2-year cycles (1999-2018) of the continuous NHANES. NHANES data is a nationally representative survey that uses a complex multistage probability sample to create a representative sample of the noninstitutionalized civilian US population. NHANES is the flagship survey for assessing the diets of the US population. The response rates to the survey differ by year, but were $62.8 \%$ in $2017-2018$ and $82 \%$ in 1999-2000 [17]. The National Center for Health Statistics (NCHS) obtained Institutional Review Board approval and informed consent was obtained from all subjects; the data have subsequently been made freely available for public use [18].

\subsection{Dietary Recall Data}

For primary analyses, dietary data came from a single 24-h dietary recall conducted in-person. A single 24-h recall for a large sample size will lead to an unbiased estimate of population-level average intakes [19]. Respondents reported the types and amounts of all foods and beverages consumed in the preceding $24 \mathrm{~h}$, from midnight to midnight in an in-person dietary recall using a computerized assisted Automated Multiple Pass Method. The method probes for commonly forgotten foods and queries detailed information on the amounts of foods consumed using common reference units and examples [20]. For young children $(<6 \mathrm{y})$ the parent was the primary respondent; for children $6-11$ y, the child was the primary respondent, but a parent/guardian was present and could assist. For children aged $\geq 12 y$, the child was the primary source of dietary recall, but could be assisted by an adult. Dietary recalls were conducted in either Spanish or English.

\subsection{Analysis Approach}

Secondary analyses examined the proportion of the population consuming vitamin $\mathrm{C}$ levels below pre-specified thresholds (e.g., Estimated Average Requirement). Because a single 24-h recall (or even the average of multiple recalls) cannot reliably estimate the population distribution of intakes we used the National Cancer Institute (NCI) method to estimate the proportion of the population consuming intakes below this threshold. As this method requires a subset of participants to have a second 24-h recall we used data from 2003-onwards for these analyses (1999-2002 public-use data do not include a second dietary recall). The second dietary recall is conducted over the telephone after the initial 24-h recall.

To examine trends in vitamin C intake stratified by food/beverage source (hereafter referred to as source) data were broken into five mutually exclusive groups: $100 \%$ fruit juice, whole fruit, vegetables, fruit drinks and other sources. These categories were created based on preliminary analyses examining the top sources of vitamin $C$ in the diet. Each category was defined based on the prefix code within the Food and Nutrient Database for Dietary Studies [21]. For example, foods starting with a "7" were identified as vegetables (including vegetable juices), those starting as "611", "642" and "643" were identified as 
100\% fruit juice, and "9252", "9253", "9255" among others were identified as fruit drinks. Because mixed dishes may contain many of these food groups (e.g., apple pie or mixed meat dishes would be classified as "other" but would include vitamin C from apples and vegetables respectively). As such, these categories should be considered as generally representative and not a full accounting of the total contribution of fruits, vegetables or fruit juices. It was not possible to use the What We Eat in America food categories as this database is only available from 2005 onwards.

Analyses were conducted for the total population age $\geq 1 \mathrm{y}$ who had at least one valid 24-h dietary recall as defined by NCHS staff. Per standard analysis procedures of 24-h dietary recall data, there was no additional exclusion of individuals based on extremely low or high energy intakes. Pregnant and lactating women were included in the analysis of dietary recalls.

\subsection{Biochemical Indicators of Vitamin C Intake}

Secondary analyses compared biochemical indicators of vitamin $C$ measured in sera for 2003-2006 (pooled from two cycles) and 2017-2018 among individuals with valid serum vitamin C levels (age $\geq 6 \mathrm{y}$ ) participating in the laboratory assessment of NHANES. Pregnant women were excluded. The protocols for data collection and analyses were generally comparable across the years and included both fasted and unfasted samples [22,23]. Consistent with prior analyses, vitamin $C$ levels were transformed using a square-root transformation and back-transformed for data presentation [24,25]. Because multiple factors can influence vitamin $C$ measurements and these factors may have changed over time (e.g., decreasing smoking and increasing obesity) both crude and adjusted analyses were conducted [26,27]. Adjusted analyses included age (using unrestricted splines), gender, race/ethnicity, family income-to-poverty ratio (with a missing indicator), measured body mass index (BMI) and an indicator of smoking status derived from serum cotinine levels. As analyses included both children and adults, a combined BMI measure was created that grouped people into an underweight ( $<18.5$ for adults; $<5 \%$ ile for children), healthy weight (18.5-24.9; 5-84.9\% ile), overweight (25-29.9; 85-94.9\% ile) and obese ( $\geq 30 ; \geq 95 \%$ ile) based on standard cut-points for adults and percentiles from the Centers for Disease Control and Prevention (CDC) growth charts for children. Current smokers were defined as individuals with a serum cotinine level $\geq 10 \mathrm{ng} / \mathrm{mL}$ consistent with the prior literature, with all others considered non-smokers [24]. Serum cotinine data was used instead of self-report data as consistent self-report data was not available for children/adolescents and smoking status is very strongly associated with serum vitamin $C$ levels. Use of supplements and consumption of vitamin $C$ in the diet was not adjusted for as they are on the causal pathway in assessing any potential trends in vitamin $C$ levels.

\subsection{Statistical Analyses}

For each 2-year cycles, the survey-weighted mean and corresponding $95 \%$ confidence interval of average vitamin $C$ intakes from sources was calculated. A survey-weighted linear regression model was then used to determine if there was a significant linear trend in intakes of vitamin C. Analyses were repeated for age/sex groups defined by the Dietary Reference Intakes (DRI) publication [28]. Analyses by source of vitamin C were conducted in a similar manner and for the overall population, children and adolescents (age 1-18 y) and adults ( $\geq 19$ y) separately. The NCI method was used to estimate the proportion of the population both overall and by age/sex population sub-groups from the DRI [29]. For analyses of serum vitamin $C$ both crude and adjusted models were implemented using survey-weighted linear regression models of the square-root transformed serum vitamin $C$ level, with results backtransformed for interpretation. A complete case analysis approach was used for the serum vitamin $\mathrm{C}$ analysis (with missing indicators for BMI and poverty status). Given the large sample size and number of statistical tests being employed a two-sided $\alpha$-level of 0.01 was used to determine statistical significance. Primary analyses used Stata 16.0 (College Station, TX, USA) and implementation of the NCI method was done in SAS 9.4 (SAS Institute Inc., 
Cary, NC, USA) using macros developed and publicly available on the NCI website [30]. All analyses appropriately accounted for the complex survey design of NHANES data.

\section{Results}

For primary analyses, the sample size was 84,902 (range across survey cycles 7284 to 9322. Overall sample characteristics across the 20-year study period are shown in Table 1, which as per design of the survey show the data are representative of the US population. The mean vitamin $C$ levels are shown for each socio-demographic group for reference. Young children and adolescents and younger adults had the highest average intakes of vitamin $C$, and males consumed about $15.2 \%$ more vitamin C (though this difference is explained by energy intakes; on an energy-adjusted basis, female participants consumed more vitamin C [ $87.4 \mathrm{mg} / \mathrm{d}$ vs. $74.8 \mathrm{mg} / \mathrm{d}$ per $2000 \mathrm{kcal}])$. By race/ethnicity, the non-Hispanic white population consumed the least vitamin $C$ and the Mexican-American population consumed the most, though these differences are largely attributable to differences in the underlying age distributions of the different populations. The highest income individuals consumed marginally more vitamin $C$ than other groups, but there was no clear gradient by income.

Table 1. Population characteristics, 1999-2018.

\begin{tabular}{|c|c|c|c|}
\hline & $N$ & Weighted \% & Mean Vitamin C, mg/d (95\% CI) \\
\hline Total & 84,902 & 100.0 & $85(83,86)$ \\
\hline \multicolumn{4}{|l|}{ Age group } \\
\hline $1-3$ & 6605 & 4.0 & $88(85,91)$ \\
\hline $4-8$ & 8534 & 6.8 & $81(78,84)$ \\
\hline $9-13$ & 9219 & 6.9 & $75(73,78)$ \\
\hline $14-18$ & 9976 & 7.1 & $81(77,84)$ \\
\hline $19-30$ & 11,211 & 17.0 & $87(84,90)$ \\
\hline $31-50$ & 16,322 & 27.6 & $86(83,89)$ \\
\hline $51-70$ & 15,214 & 22.4 & $85(83,88)$ \\
\hline$\geq 71$ & 7821 & 8.1 & $85(82,88)$ \\
\hline \multicolumn{4}{|l|}{$\overline{\text { Sex }}$} \\
\hline Female & 43,138 & 51.2 & $79(77,80)$ \\
\hline Male & 41,764 & 48.8 & $91(89,93)$ \\
\hline \multicolumn{4}{|l|}{ Race/ethnicity } \\
\hline Non-Hispanic White & 32,117 & 65.3 & $80(78,82)$ \\
\hline Non-Hispanic Black & 20,007 & 12.0 & $94(92,97)$ \\
\hline Mexican-American & 18,577 & 9.8 & $95(91,98)$ \\
\hline Other Hispanic & 6801 & 5.7 & $92(89,96)$ \\
\hline Other race/mixed race & 7400 & 7.1 & $87(83,90)$ \\
\hline \multicolumn{4}{|c|}{ Family income-to-poverty ratio a } \\
\hline$<1.00$ [lower income] & 20,621 & 16.2 & $85(82,88)$ \\
\hline $1.00-1.99$ & 20,957 & 20.1 & $80(77,83)$ \\
\hline $2.00-3.99$ & 19,880 & 26.5 & $81(79,83)$ \\
\hline$\geq 4.00$ [higher income] & 16,625 & 30.2 & $90(87,93)$ \\
\hline Missing & 6819 & 6.9 & $87(94,91)$ \\
\hline
\end{tabular}

\footnotetext{
${ }^{a}$ Interpreted as ratio of family income to the federal poverty guidelines. In 2018 , the federal poverty
} level for a family of four in the contiguous United States was $\$ 25,100$.

Trends in mean vitamin C intakes over the 20-year period are shown in Table 2. On average, vitamin C levels decreased by about $2.3 \mathrm{mg} / \mathrm{d}$ for each 2-year period or by $22.6 \%$ overall ( $p$-value for trend $<0.001)$. Declines were particularly dramatic among children 1-3 y (change from 1999-2000 to 2017-2018: -32.1\%), 14-18y females $(-39.6 \%)$, $14-18 y$ males $(-33.6 \%)$, women $51-70$ y $(-30.7 \%)$, and men $51-70$ y $(-25.7 \%)$. The only subgroups not experiencing a statistically significant decline were 9-13y males and females, 31-50 y females and older adults ( $\geq 71 \mathrm{y}$ ). Because other changes in the composition of the population can affect trends in dietary intakes, sensitivity analyses evaluated overall trends adjusted for age, sex, race/ethnicity and energy intakes. There was no observable difference between adjusted and unadjusted results (see Table 3). 
Table 2. Trends in population mean vitamin C consumption from foods and beverages (mg/d) overall and by Dietary Reference Intake age and sex groups, $1999-2018$.

\begin{tabular}{|c|c|c|c|c|c|c|c|c|c|c|c|c|}
\hline & \multicolumn{11}{|c|}{$\begin{array}{c}\text { Vitamin C mg/d } \\
(95 \% \mathrm{CI})\end{array}$} & \multirow{2}{*}{$\begin{array}{l}p \text {-Value for } \\
\text { Trend }\end{array}$} \\
\hline & EAR (mg) & 1999-2000 & 2001-2002 & 2003-2004 & 2005-2006 & $2007-2008$ & 2009-2010 & 2011-2012 & 2013-2014 & 2015-2016 & $2017-2018$ & \\
\hline $\begin{array}{c}\text { Total } \\
\text { population } \\
\text { (age } \geq 1 \mathrm{y} \text { ) } \\
\text { Age/sex } \\
\text { groups }\end{array}$ & - & $\begin{array}{c}97 \\
(90,105)\end{array}$ & $\begin{array}{c}94 \\
(87,100)\end{array}$ & $\begin{array}{c}88 \\
(83,94)\end{array}$ & $\begin{array}{c}86 \\
(83,89)\end{array}$ & $\begin{array}{c}84 \\
(77,91)\end{array}$ & $\begin{array}{c}86 \\
(84,89)\end{array}$ & $\begin{array}{c}83 \\
(77,90)\end{array}$ & $\begin{array}{c}78 \\
(75,80)\end{array}$ & $\begin{array}{c}77 \\
(72,81)\end{array}$ & $\begin{array}{c}75 \\
(71,80)\end{array}$ & $<0.001$ \\
\hline $1-3$ & 22 & $\begin{array}{c}109 \\
(95,123)\end{array}$ & $\begin{array}{c}92 \\
(86,98)\end{array}$ & $\begin{array}{c}105 \\
(93,116)\end{array}$ & $\begin{array}{c}84 \\
(75,94)\end{array}$ & $\begin{array}{c}102 \\
(91,112)\end{array}$ & $\begin{array}{c}81 \\
(74,88)\end{array}$ & $\begin{array}{c}85 \\
(71,99)\end{array}$ & $\begin{array}{c}81 \\
(75,88)\end{array}$ & $\begin{array}{c}68 \\
(61,76)\end{array}$ & $\begin{array}{c}74 \\
(63,84)\end{array}$ & $<0.001$ \\
\hline $4-8$ & 39 & $\begin{array}{c}93 \\
(85,101)\end{array}$ & $\begin{array}{c}80 \\
(71,90)\end{array}$ & $\begin{array}{c}91 \\
(83,99)\end{array}$ & $\begin{array}{c}80 \\
(70,91)\end{array}$ & $\begin{array}{c}86 \\
(77,94)\end{array}$ & $\begin{array}{c}79 \\
(71,86)\end{array}$ & $\begin{array}{c}84 \\
(76,92)\end{array}$ & $\begin{array}{c}72 \\
(66,79)\end{array}$ & $\begin{array}{c}67 \\
(55,78)\end{array}$ & $\begin{array}{c}79 \\
(68,90)\end{array}$ & $<0.001$ \\
\hline $9-13$, female & 56 & $\begin{array}{c}77 \\
(68,87)\end{array}$ & $\begin{array}{c}82 \\
(71,92)\end{array}$ & $\begin{array}{c}84 \\
(74,94)\end{array}$ & $\begin{array}{c}76 \\
(66,86)\end{array}$ & $\begin{array}{c}67 \\
(53,81)\end{array}$ & $\begin{array}{c}73 \\
(62,85)\end{array}$ & $\begin{array}{c}69 \\
(58,80)\end{array}$ & $\begin{array}{c}64 \\
(57,72)\end{array}$ & $\begin{array}{c}64 \\
(59,68)\end{array}$ & $\begin{array}{c}80 \\
(62,98)\end{array}$ & 0.08 \\
\hline 9-13, male & 63 & $\begin{array}{c}81 \\
(68,94)\end{array}$ & $\begin{array}{c}81 \\
(69,92)\end{array}$ & $\begin{array}{c}77 \\
(67,87)\end{array}$ & $\begin{array}{c}73 \\
(63,83)\end{array}$ & $\begin{array}{c}87 \\
(73,101)\end{array}$ & $\begin{array}{c}68 \\
(55,81)\end{array}$ & $\begin{array}{c}80 \\
(70,90)\end{array}$ & $\begin{array}{c}80 \\
(69,92)\end{array}$ & $\begin{array}{c}73 \\
(61,84)\end{array}$ & $\begin{array}{c}70 \\
(56,83)\end{array}$ & 0.21 \\
\hline $14-18$, female & 60 & $\begin{array}{c}91 \\
(84,99)\end{array}$ & $\begin{array}{c}78 \\
(63,92)\end{array}$ & $\begin{array}{c}77 \\
(68,86)\end{array}$ & $\begin{array}{c}74 \\
(64,84)\end{array}$ & $\begin{array}{c}77 \\
(65,89)\end{array}$ & $\begin{array}{c}73 \\
(62,85)\end{array}$ & $\begin{array}{c}59 \\
(54,64)\end{array}$ & $\begin{array}{c}65 \\
(54,76)\end{array}$ & $\begin{array}{c}66 \\
(54,79)\end{array}$ & $\begin{array}{c}55 \\
(46,63)\end{array}$ & $<0.001$ \\
\hline 19-30, female & 60 & $\begin{array}{c}87 \\
(76,97)\end{array}$ & $\begin{array}{c}88 \\
(77,98)\end{array}$ & $\begin{array}{c}82 \\
(70,94)\end{array}$ & $\begin{array}{c}78 \\
(71,85)\end{array}$ & $\begin{array}{c}80 \\
(66,95)\end{array}$ & $\begin{array}{c}80 \\
(72,89)\end{array}$ & $\begin{array}{c}80 \\
(69,92)\end{array}$ & $\begin{array}{c}71 \\
(66,75)\end{array}$ & $\begin{array}{c}75 \\
(65,85)\end{array}$ & $\begin{array}{c}71 \\
(62,80)\end{array}$ & 0.002 \\
\hline 19-30, male & 75 & $\begin{array}{c}100 \\
(84,116)\end{array}$ & $\begin{array}{c}123 \\
(93,154)\end{array}$ & $\begin{array}{c}94 \\
(81,108)\end{array}$ & $\begin{array}{c}106 \\
(90,121)\end{array}$ & $\begin{array}{c}96 \\
(82,111)\end{array}$ & $\begin{array}{c}101 \\
(86,116)\end{array}$ & $\begin{array}{c}96 \\
(78,114)\end{array}$ & $\begin{array}{c}87 \\
(75,100)\end{array}$ & $\begin{array}{c}87 \\
(75,99)\end{array}$ & $\begin{array}{c}71 \\
(61,81)\end{array}$ & $<0.001$ \\
\hline $31-50$, female & 60 & $\begin{array}{c}87 \\
(72,102)\end{array}$ & $\begin{array}{c}82 \\
(69,96)\end{array}$ & $\begin{array}{c}85 \\
(73,96)\end{array}$ & $\begin{array}{c}76 \\
(63,89)\end{array}$ & $\begin{array}{c}75 \\
(63,87)\end{array}$ & $\begin{array}{c}80 \\
(71,88)\end{array}$ & $\begin{array}{c}76 \\
(68,84)\end{array}$ & $\begin{array}{c}74 \\
(66,82)\end{array}$ & $\begin{array}{c}73 \\
(64,82)\end{array}$ & $\begin{array}{c}74 \\
(67,82)\end{array}$ & 0.028 \\
\hline $31-50$, male & 75 & $\begin{array}{c}109 \\
(96,122)\end{array}$ & $\begin{array}{c}107 \\
(89,125)\end{array}$ & $\begin{array}{c}99 \\
(84,115)\end{array}$ & $\begin{array}{c}95 \\
(87,103)\end{array}$ & $\begin{array}{c}95 \\
(83,108)\end{array}$ & $\begin{array}{c}97 \\
(85,108)\end{array}$ & $\begin{array}{c}86 \\
(73,99)\end{array}$ & $\begin{array}{c}76 \\
(70,82)\end{array}$ & $\begin{array}{c}84 \\
(74,95)\end{array}$ & $\begin{array}{c}85 \\
(72,98)\end{array}$ & $<0.001$ \\
\hline $51-70$, female & 60 & $\begin{array}{c}101 \\
(89,112)\end{array}$ & $\begin{array}{c}94 \\
(87,101)\end{array}$ & $\begin{array}{c}78 \\
(68,87)\end{array}$ & $\begin{array}{c}78 \\
(71,85)\end{array}$ & $\begin{array}{c}82 \\
(72,91)\end{array}$ & $\begin{array}{c}91 \\
(78,105)\end{array}$ & $\begin{array}{c}76 \\
(69,82)\end{array}$ & $\begin{array}{c}75 \\
(69,80)\end{array}$ & $\begin{array}{c}74 \\
(66,82)\end{array}$ & $\begin{array}{c}70 \\
(62,77)\end{array}$ & $<0.001$ \\
\hline 51-70, male & 75 & $\begin{array}{c}109 \\
(96,122)\end{array}$ & $\begin{array}{c}101 \\
(92,110)\end{array}$ & $\begin{array}{c}87 \\
(80,94)\end{array}$ & $\begin{array}{c}98 \\
(89,108)\end{array}$ & $\begin{array}{c}85 \\
(75,95)\end{array}$ & $\begin{array}{c}91 \\
(84,98)\end{array}$ & $\begin{array}{c}99 \\
(83,114)\end{array}$ & $\begin{array}{c}83 \\
(75,90)\end{array}$ & $\begin{array}{c}83 \\
(73,93)\end{array}$ & $\begin{array}{c}81 \\
(72,90)\end{array}$ & $<0.001$ \\
\hline$\geq 71$, female & 60 & $\begin{array}{c}94 \\
(85,103)\end{array}$ & $\begin{array}{c}85 \\
(75,94)\end{array}$ & $\begin{array}{c}83 \\
(76,89)\end{array}$ & $\begin{array}{c}81 \\
(75,88)\end{array}$ & $\begin{array}{c}74 \\
(68,80)\end{array}$ & $\begin{array}{c}78 \\
(72,85)\end{array}$ & $\begin{array}{c}81 \\
(71,92)\end{array}$ & $\begin{array}{c}85 \\
(78,92)\end{array}$ & $\begin{array}{c}76 \\
(64,87)\end{array}$ & $\begin{array}{c}75 \\
(63,87)\end{array}$ & 0.031 \\
\hline
\end{tabular}


Table 2. Cont.

\begin{tabular}{|c|c|c|c|c|c|c|c|c|c|c|c|c|}
\hline & \multicolumn{11}{|c|}{$\begin{array}{l}\text { Vitamin C mg/d } \\
(95 \% \mathrm{CI})\end{array}$} & \multirow{2}{*}{$\begin{array}{c}p \text {-Value for } \\
\text { Trend }\end{array}$} \\
\hline & EAR (mg) & 1999-2000 & 2001-2002 & 2003-2004 & 2005-2006 & $2007-2008$ & 2009-2010 & 2011-2012 & 2013-2014 & 2015-2016 & $2017-2018$ & \\
\hline$\geq 71$, male & 75 & $\begin{array}{c}112 \\
(99,125)\end{array}$ & $\begin{array}{c}90 \\
(82,99)\end{array}$ & $\begin{array}{c}87 \\
(79,96)\end{array}$ & $\begin{array}{c}98 \\
(84,112)\end{array}$ & $\begin{array}{c}86 \\
(78,95)\end{array}$ & $\begin{array}{c}89 \\
(75,103)\end{array}$ & $\begin{array}{c}83 \\
(69,96)\end{array}$ & $\begin{array}{c}97 \\
(85,109)\end{array}$ & $\begin{array}{c}88 \\
(74,101)\end{array}$ & $\begin{array}{c}85 \\
(71,99)\end{array}$ & 0.06 \\
\hline
\end{tabular}

EAR refers to Estimated Average Requirement.

Table 3. Sensitivity analyses for overall trend adjusted for covariates.

\begin{tabular}{|c|c|c|c|c|c|c|c|c|c|c|c|}
\hline & \multicolumn{10}{|c|}{ Vitamin C mg/d (95\% CI) } & \multirow{2}{*}{$\begin{array}{l}p \text {-Value for } \\
\text { Trend }\end{array}$} \\
\hline & 1999-2000 & 2001-2002 & 2003-2004 & 2005-2006 & 2007-2008 & 2009-2010 & 2011-2012 & 2013-2014 & 2015-2016 & 2017-2018 & \\
\hline Unadjusted & $\begin{array}{c}97 \\
(90,105)\end{array}$ & $\begin{array}{c}94 \\
(87,100)\end{array}$ & $\begin{array}{c}88 \\
(83,94)\end{array}$ & $\begin{array}{c}86 \\
(83,89)\end{array}$ & $\begin{array}{c}84 \\
(77,91)\end{array}$ & $\begin{array}{c}86 \\
(84,89)\end{array}$ & $\begin{array}{c}83 \\
(77,90)\end{array}$ & $\begin{array}{c}78 \\
(75,80)\end{array}$ & $\begin{array}{c}77 \\
(72,81)\end{array}$ & $\begin{array}{c}75 \\
(71,80)\end{array}$ & $<0.001$ \\
\hline $\begin{array}{c}\text { Age- } \\
\text { adjusted }\end{array}$ & $\begin{array}{c}97 \\
(90,105)\end{array}$ & $\begin{array}{c}94 \\
(88,100)\end{array}$ & $\begin{array}{c}88 \\
(83,94)\end{array}$ & $\begin{array}{c}86 \\
(83,90)\end{array}$ & $\begin{array}{c}84 \\
(77,91)\end{array}$ & $\begin{array}{c}86 \\
(84,89)\end{array}$ & $\begin{array}{c}83 \\
(77,90)\end{array}$ & $\begin{array}{c}78 \\
(75,80)\end{array}$ & $\begin{array}{c}76 \\
(72,81)\end{array}$ & $\begin{array}{c}75 \\
(71,79)\end{array}$ & $<0.001$ \\
\hline $\begin{array}{l}\text { Age/sex- } \\
\text { adjusted }\end{array}$ & $\begin{array}{c}97 \\
(90,105)\end{array}$ & $\begin{array}{c}94 \\
(88,100)\end{array}$ & $\begin{array}{c}88 \\
(83,94)\end{array}$ & $\begin{array}{c}86 \\
(83,90)\end{array}$ & $\begin{array}{c}84 \\
(77,92)\end{array}$ & $\begin{array}{c}86 \\
(84,89)\end{array}$ & $\begin{array}{c}83 \\
(77,90)\end{array}$ & $\begin{array}{c}77 \\
(75,80)\end{array}$ & $\begin{array}{c}76 \\
(72,81)\end{array}$ & $\begin{array}{c}75 \\
(71,80)\end{array}$ & $<0.001$ \\
\hline $\begin{array}{l}\text { Age/sex/race } \\
\text { adjusted }\end{array}$ & $\begin{array}{c}98 \\
(91,105)\end{array}$ & $\begin{array}{c}94 \\
(89,100)\end{array}$ & $\begin{array}{c}89 \\
(84,94)\end{array}$ & $\begin{array}{c}87 \\
(83,90)\end{array}$ & $\begin{array}{c}85 \\
(78,91)\end{array}$ & $\begin{array}{c}86 \\
(84,89)\end{array}$ & $\begin{array}{c}83 \\
(77,89)\end{array}$ & $\begin{array}{c}77 \\
(75,80)\end{array}$ & $\begin{array}{c}76 \\
(71,80)\end{array}$ & $\begin{array}{c}74 \\
(70,79)\end{array}$ & $<0.001$ \\
\hline $\begin{array}{l}\text { Energy- } \\
\text { adjusted }\end{array}$ & $\begin{array}{c}94 \\
(87,101)\end{array}$ & $\begin{array}{c}90 \\
(84,96)\end{array}$ & $\begin{array}{c}84 \\
(78,89)\end{array}$ & $\begin{array}{c}83 \\
(79,86)\end{array}$ & $\begin{array}{c}83 \\
(76,90)\end{array}$ & $\begin{array}{c}85 \\
(82,87)\end{array}$ & $\begin{array}{c}80 \\
(74,87)\end{array}$ & $\begin{array}{c}76 \\
(74,78)\end{array}$ & $\begin{array}{c}76 \\
(71,80)\end{array}$ & $\begin{array}{c}73 \\
(69,77)\end{array}$ & $<0.001$ \\
\hline $\begin{array}{c}\text { Fully- } \\
\text { adjusted }\end{array}$ & $\begin{array}{c}94 \\
(88,101)\end{array}$ & $\begin{array}{c}91 \\
(85,96)\end{array}$ & $\begin{array}{c}84 \\
(80,89)\end{array}$ & $\begin{array}{c}83 \\
(79,87)\end{array}$ & $\begin{array}{c}83 \\
(77,89)\end{array}$ & $\begin{array}{c}85 \\
(82,87)\end{array}$ & $\begin{array}{c}79 \\
(73,86)\end{array}$ & $\begin{array}{c}75 \\
(73,78)\end{array}$ & $\begin{array}{c}74 \\
(70,79)\end{array}$ & $\begin{array}{c}72 \\
(67,76)\end{array}$ & $<0.001$ \\
\hline
\end{tabular}

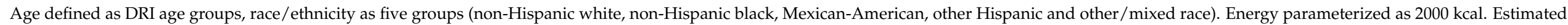
as adjusted marginal means. 
Trends in sources of vitamin $\mathrm{C}$ are provided in Figure 1. Overall (Figure 1A), the decline in vitamin $C$ intakes appears to be driven by decreases in vitamin C from $100 \%$ fruit juice and fruit drinks. In 1999-2000, 100\% fruit juice was the second leading source of vitamin C (just below fruit), but in 2017-2018, it was the fourth leading source of vitamin C, declining by nearly half $(-48.4 \%)$. At the same time, vitamin $C$ from vegetables was generally unchanged and vitamin $C$ from whole fruit increased by about $25.8 \%$. The contribution of fruit drinks to vitamin $C$ intakes also declined dramatically by more than half $(-50.7 \%)$. Vitamin $C$ from other sources did not materially change. Some differences in trends by sources were observed when data were stratified by age. Among children/adolescents (Figure 1B), the contribution of whole fruit to vitamin $C$ intakes increased, whereas no increase was observed for adults (Figure 1C). While the contribution of juice declined in both groups, the decline was more pronounced among adults. For children/adolescents there was a modest decline in vitamin $C$ from vegetables and some suggestion of the same for adults, but not statistically significant.

\subsection{EAR Results}

For the overall population, the proportion of the population below the EAR increased from $38.3 \%$ to $47.4 \%$ from 2003-2004 to 2017-2018, a 24\% relative increase (Figure 2A). Figure 2B shows the proportion of the population below the EAR by age/sex group in 20032004 compared to 2017-2018. The most marked increase in the proportion below the EAR was among the youngest (1-3 y), females 14-18 y, males 14-18 y, males 19-30 y, males 31-50 $y$ and older males. The only sub-group with a decrease in the proportion below the EAR were children $4-8 \mathrm{y}$. 


\section{A: Total population}

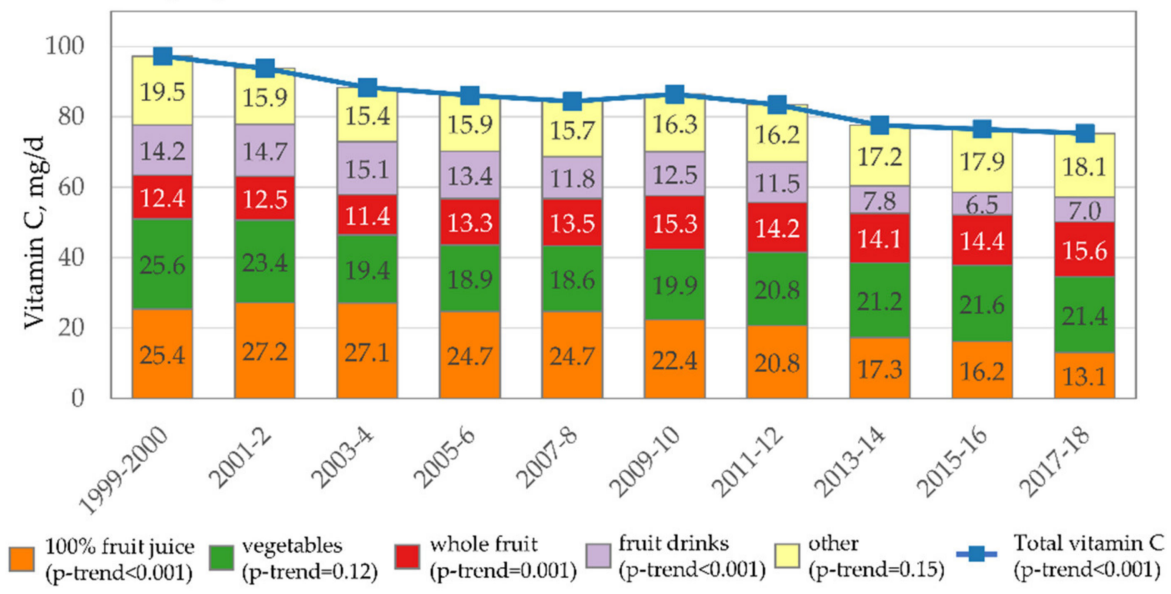

B: Children and adolescents (1-18y)

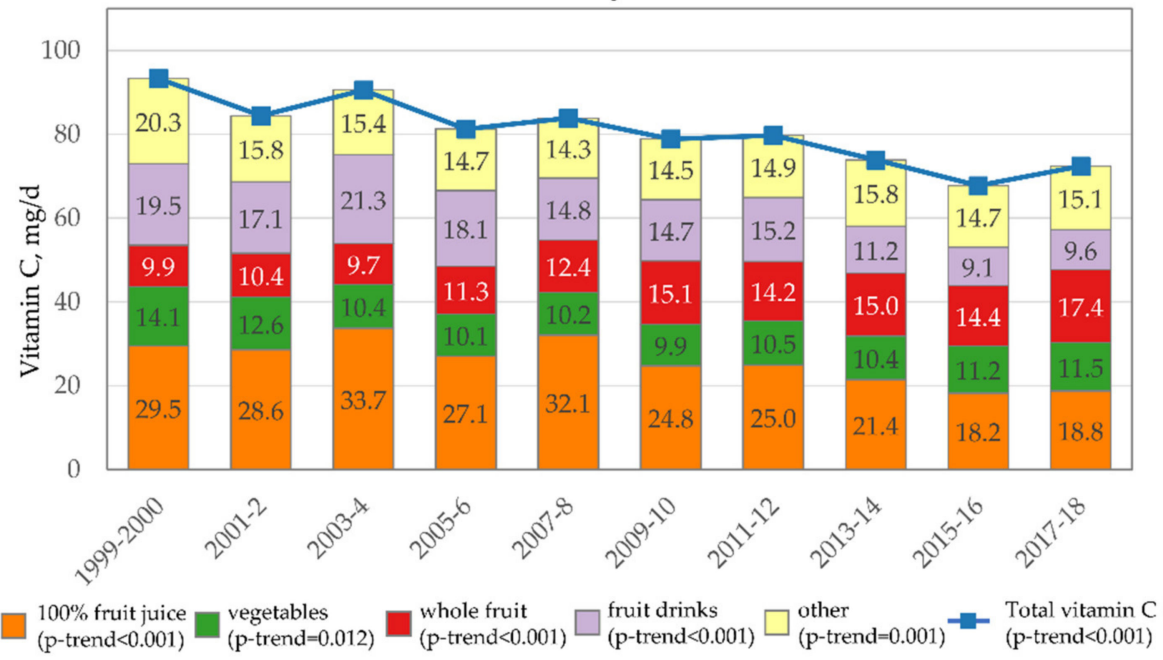

C: Adults ( $\geq 19 y)$

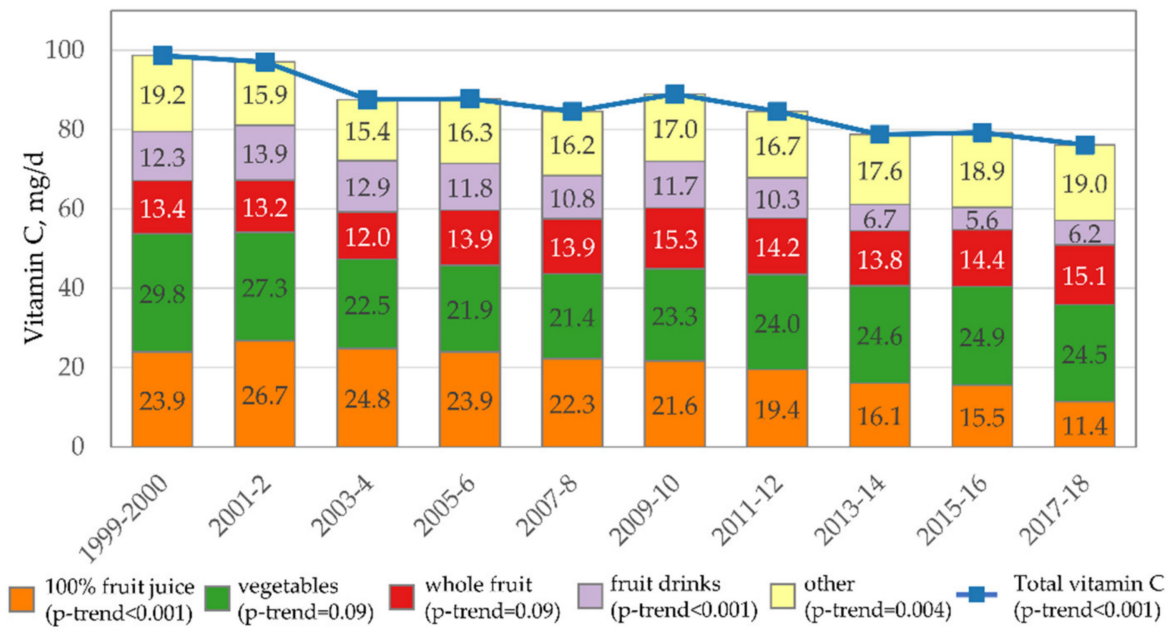

Figure 1. Trend in vitamin $C$ intakes overall and by specific food/beverage source overall (A), among children/adolescents 1-18 y (B), and adults $\geq 19$ y (C), 1999-2018. 


\section{A: Proportion below the EAR by year}

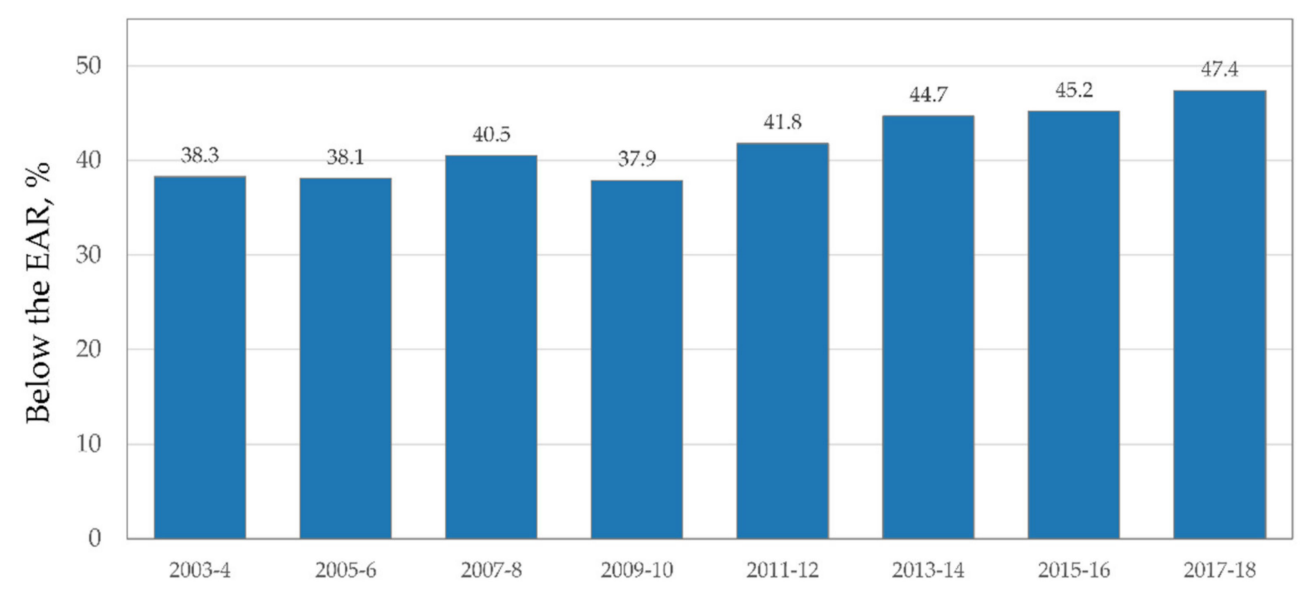

B: Proportion below EAR by age/sex group: $2003-4$ vs. 2017-18

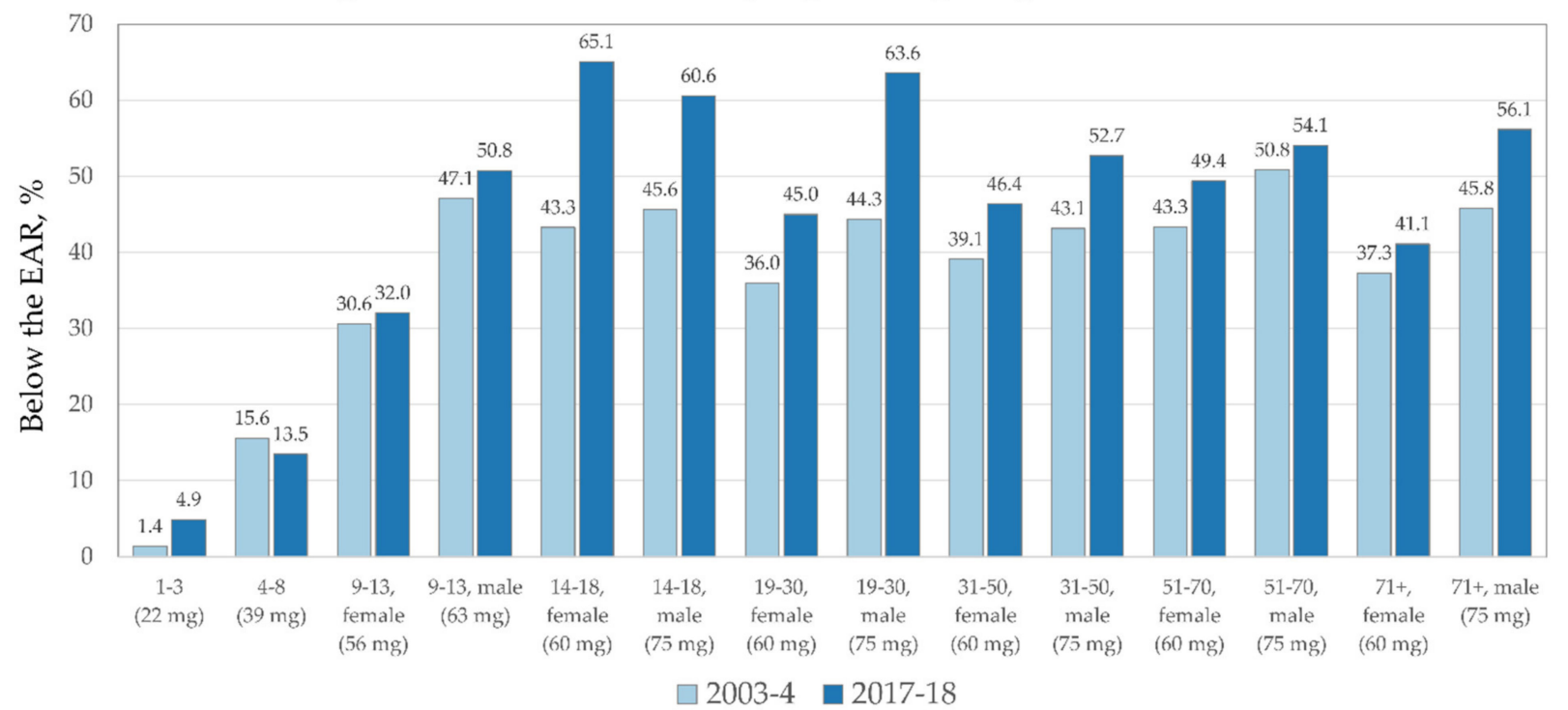

Figure 2. Trends in proportion of population below the Estimated Average Requirement (EAR) overall (A) and by age/sex group (B).

\subsection{Trends Based on Biomarkers of Vitamin C}

A total of 20,675 individuals age $\geq 6$ y (6696 in 2017-2018 and 13,979 in 2003-2006) were included in the analysis of serum vitamin $C$ levels. The results of the serum vitamin $\mathrm{C}$ analysis diet are shown in Figure 3. Overall, there was a non-significant decline in serum vitamin C levels in fully-adjusted analyses of approximately 4.8\% from 2003-2006 to 20172018. There was little suggestion of a difference in serum vitamin C levels for children and adolescents. For adults, there was some evidence of a decrease (declined by approximately $5.5 \%$ ), but like the overall analysis, this difference was not statistically significant. 


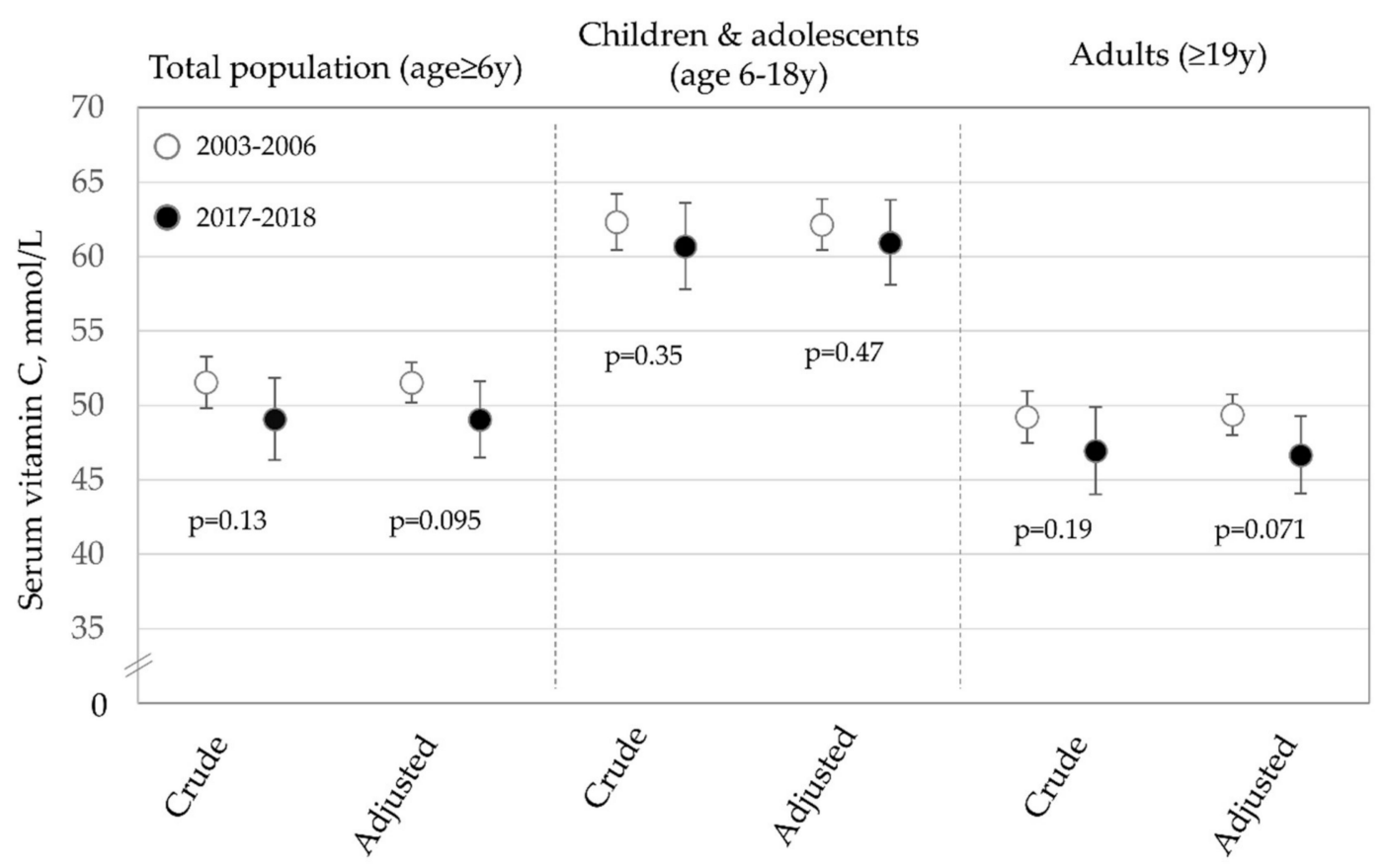

Figure 3. Mean serum vitamin C concentrations in 2003-2006 compared to 2017-2018 overall, among children/adolescents and adults (Adjusted model includes covariates for age group, race/ethnicity, sex, BMI, family income-to-poverty ratio and serum cotinine levels. Overall sample size is 20,675, 6821 for children/adolescents and 13,854 for adults).

\section{Discussion}

In this large nationally-representative survey of more than 84,000 participants with high-quality dietary data collected over a 20-year period we observed a $22.6 \%$ decrease in vitamin $C$ intakes. This decrease was observed amongst all but a limited number of age/sex groups and appears to be driven by reduced contribution of vitamin $C$ from $100 \%$ fruit juice and no marked change in vitamin $C$ from vegetables. Vitamin $C$ from whole fruits increased for children/adolescents, but not adults. From 2003-2004 to 2017-2018, the proportion of individuals with intakes below the EAR increased from $38.3 \%$ to $47.4 \%$. Analyses of serum vitamin $C$ observed a non-significant decrease in vitamin $C$ levels, particularly for adults, but the proportional decline was less than was observed for diet.

While far from optimal, prior population-based studies have shown that the US-diet has modestly improved over the past 15-20 years. Notable changes include increased whole grain, fiber, polyunsaturated fat and whole fruit consumption and decreased consumption of added sugars and solid fats [31]. Fiber $(+16.6 \%$ for adults; $+25.3 \%$ for children/adolescents) and calcium ( $+20.1 \%$ for adults; $+21.2 \%$ for children), two nutrients of public health concern, increased among adults and children from 1999-2012 and 1999-2016, respectively [31,32]. Vitamin D intakes have not meaningfully changed among adults [31] and no major trend was observed for potassium. The current finding of fairly-dramatic decreases in vitamin $C$ intakes in the context of improving diet quality overall represents somewhat of a paradox.

One possible explanation for these findings is the reduced consumption of $100 \%$ fruit juices. Our results show that in 1999-2000, about $26 \%$ of vitamin C consumed in the diet came from $100 \%$ fruit juice, declining to $17 \%$ by $2017-2018$. This finding is consistent with a large body of research demonstrating decreased consumption of $100 \%$ fruit juice [31-35]. To date, studies documenting changes in fruit juice consumption have not formally assessed the potential impact on nutrient intakes. While many professional organizations recommend whole fruit over $100 \%$ fruit juice, juice is a beverage and is more practically replaced by another beverage such as water, juice drinks and other beverages or none-at-all rather than a direct exchange for whole fruit [36,37]. Outside of milk (where 
consumption has dropped precipitously), 100\% fruit juices tend to have a very high nutrient density compared to other beverages [38].

While not directly quantifiable, much of these consumption changes may be due to messaging around the sugar content of juice and purported links between sugar and adverse health outcomes [39] which have also translated to recomendations from the American Academy of Pediatrics suggesting limiting juice intake in children to certain age-specific levels [40]. The observed change in whole fruit intake is less consistent on the population level with children increasing intake far more than adults. This difference may be due to national efforts such as revamping the school lunch program and initiatives like "Let's Move" and "Play 60" ad campaigns that are specifically targeted towards children. Curiously, trends in whole fruit consumption show increasing intakes among US adults, at least through 2011-2012. This suggests that the increase in whole fruit was not for vitamin $C$ rich fruits (e.g., citrus, berries or tropical fruits) and may have been for fruits with relatively little vitamin C (e.g., apples or bananas), the latter of which are amongst the most commonly consumed fruits in the US [41]. This observation highlights the need to conduct detailed analyses that not only examine trends in dietary intakes by broad food sources but using as fine-resolution as possible to truly understand the dietary changes being made in the population.

While the changes in vitamin $C$ sources from juice and whole fruit were compelling it is worth noting that we observed no marked change in vitamin $C$ contributions from vegetables, which is consistent with relatively flat consumption of vegetables over this study period. For children, we observed a modest trend towards less vitamin $C$ from vegetables and for adults there was some suggestion of such a trend ( $p$-value for trend $=0.09$ ). The generally flat intake of vegetables represents an important public health challenge as vegetables have numerous health benefits and are nutrient dense and energy sparse. Multiple campaigns and policies have been implemented to encourage vegetable consumption but there remain numerous barriers to intake. The high cost of whole fruit and vegetables, particularly fresh versions, is one often-cited barrier [42,43]. Descriptive studies show that on a per-calorie basis these are amongst the costliest foods [44] and qualitative studies show that consumers, especially those with lower-incomes, are highly attuned to their cost [45]. Beyond their direct cost, availability challenges are noteworthy, along with concerns regarding taste, quality, and time of preparation/convenience [46-48]. In part due to these barriers, individuals with higher socioeconomic status (SES) routinely consume greater amounts of whole fruits and vegetables than individuals of lower SES $[45,49]$. However, all groups consume too few fruits and vegetables, so simply eliminating disparities in intake will not be adequate to bring population-level intakes in-line with recommendations. On the other-hand numerous relatively inexpensive $100 \%$ fruit juices, are widely available. United States Department of Agriculture estimates show a median price per fruit cup equivalent of $\$ 0.32$ for frozen juices from concentrate and $\$ 0.42$ for ready-to-drink juices, compared to $\$ 0.72, \$ 1.18, \$ 0.94$ and $\$ 0.91$ for fresh, frozen, canned and dried fruits respectively [50]. In terms of food loss, the percent of consumer losses for fresh fruits and vegetables stands at 33 and 30\% respectively, compared to $13 \%$ for processed fruit (a broader category which includes juice) [51]. Future studies should be conducted to unpack the specific mechanisms informing dietary choices for vitamin $\mathrm{C}$ dense foods and beverages.

This analysis focused on vitamin $C$ from foods and beverages and deliberately did not include vitamin $C$ from supplements. This choice was made because numerous professional organizations, including the American Heart Association and American Institute for Cancer Research encourage individuals to meet their micronutrient recommendations through diet alone $[52,53]$. The Academy of Nutrition and Dietetics and the US Preventive Services Task Force do not go quite this far but both discourage supplement use for the purposes of disease prevention $[54,55]$ which stands in contrast to the most commonly reported reason reported for supplement use, which is to "improve/maintain health" [56]. An analysis of NHANES data from 1999-2012 among adults, showed the 30-day use of 
vitamin C supplements, including from multi-vitamins decreased significantly from $42 \%$ to $36 \%$ [57], but more recent data are not available. Published data on trends among children/adolescents are not available but data from 2017-2018 NHANES show only 3.0\% were taking a single ingredient vitamin C supplement and $23.8 \%$ were taking some type of multivitamin/multimineral supplement, which usually contain vitamin C [58].

It is important to consider the declines in vitamin $C$ intakes observed here in the context of dietary adequacy. The issue with declining trends in vitamin $C$ intakes from foods/beverages is not a concern of acute deficiency (e.g., scurvy), but rather on its potential impact on the immune system, skin health and collagen synthesis, nervous system and potentially on reactive oxidative species [59]. On a comparative basis the proportion below the EAR for vitamin $C$ is higher than some nutrients of public health concern. Cowan et al. show in sex-stratified analyses that the proportion of men with vitamin $C$ intakes less than the EAR (50.8\% in 2011-2014) is higher than calcium (26\%) and potassium (35\%), but much lower than vitamin D (91.5\%) [60]. For women, the proportion below the EAR was higher for vitamin $C$ than for potassium, though more women were below the EAR for calcium [60]. Another reason to monitor this decline is the connection between vitamin C intake and iron bioavailability. In the latest Dietary Guidelines for Americans, iron was identified as an under-consumed nutrient for certain groups [1] and vitamin $C$ is known to increase the bioavailability of non-heme iron [61]. As consumer interest in a "flexitarian" or "plant-based" diet has increased along with decreasing trends of some animal foods this is an issue meriting careful assessment [62,63]. Given these concerns, the decrease in mean vitamin $C$ intakes and more importantly, the increase in the proportion of individuals with intakes below the EAR is of potential concern and merits careful monitoring looking forward.

Study limitations include the use of self-reported dietary data which is subject to systematic and random errors [64]. Despite this, professionally collected 24-h recalls are considered amongst the strongest dietary assessment instruments and form the cornerstone of dietary surveillance in the US and elsewhere. Further, a single 24-h recall can provide an unbiased estimate of population average intakes. Our analysis of biochemical indicators of diet may be subject to residual confounding, via covariate measurement error or inefficient parameterization of variables, though we did adjust for variables previously shown to be strongly related to serum vitamin $C$ and both BMI and serum cotinine are objective measures not subject to self-report bias, though they may be subject to other forms of measurement error. Strengths of the study included a very large sample size, which allowed us to examine statistically reliable data by two-year cycle and present data stratified by age and sex. A further strength of the study is looking at the specific sources of changes in vitamin $C$, which allow us to start identifying the potential reasons for trends rather than simply describing them.

\section{Conclusions}

In this large population-based study we observed a dramatic decline in vitamin C intakes that appears to be driven by declines in fruit juice consumption. A modest increase in vitamin $C$ from whole fruit could not make up the gap due to declines in $100 \%$ fruit juice consumption. While at the population-level there is little risk of severe vitamin $C$ deficiency, given observed relationships and associations between vitamin $C$ intake and numerous health outcomes these trends are of potential concern. The proportion of individuals with vitamin $C$ intakes less than the EAR appear to be greater than for other nutrients of public health concern including calcium (for men) and potassium. There is tremendous need to increase fruit and vegetable consumption in the United States and multiple approaches will likely be necessary to impart these changes. Looking forward, vitamin C intakes should be continually monitored and identifying specific sub-populations at greater risk for inadequate vitamin $\mathrm{C}$ intakes could be identified. Close examinations of dietary trends for specific dietary constituents of interest beyond the overall topline trend can help us understand why the diet may be changing and identify potential strategies to improve diet. 
Author Contributions: Author contributions were as follows: M.B., M.J.D. and C.D.R. designed the research; all analyses were completed by C.D.R.; manuscript was written by C.D.R. and M.B. All authors have read and agreed to the published version of the manuscript.

Funding: This study was funded by PepsiCo Inc.

Institutional Review Board Statement: Not applicable as this study used fully de-identified publiclyavailable data which is not considered human subject research. The National Center for Health Statistics received approval from their Ethics Review Board.

Informed Consent Statement: Informed consent was obtained from all subjects involved in the study by the National Center for Health Statistics.

Data Availability Statement: All data for this project are publicly available on the National Center for Health Statistics website, available at: https:/ / wwwn.cdc.gov/nchs/nhanes/Default.aspx.

Acknowledgments: The views expressed in this manuscript are those of the authors and do not necessarily reflect the position or policy of PepsiCo Inc.

Conflicts of Interest: All authors are currently employed by PepsiCo, Inc. The authors have no additional conflicts of interest to report.

\section{References}

1. U.S. Department of Health and Human Services; U.S. Department of Agriculture. 2015-2020 Dietary Guidelines for Americans; U.S. Department of Health and Human Services; U.S. Department of Agriculture: Washington, DC, USA, 2015.

2. Hoy, M.K.; Clemens, J.C.; Martin, C.L.; Moshfegh, A.J. Fruit and Vegetable Consumption of US Adults by Level of Variety, What We Eat in America, NHANES 2013-2016. Curr. Dev. Nutr. 2020, 4, nzaa014. [CrossRef] [PubMed]

3. U.S. Department of Agriculture. Choose My Plate. Available online: https:/ / www.choosemyplate.gov/ (accessed on 30 September 2020).

4. Taylor, C.A.; Hampl, J.S.; Johnston, C.S. Low Intakes of Vegetables and Fruits, Especially Citrus Fruits, Lead to Inadequate Vitamin C Intakes among Adults. Eur. J. Clin. Nutr. 2000, 54, 573-578. [CrossRef] [PubMed]

5. National Institutes of Health. Vitamin C Fact Sheet for Consumers. Available online: https://ods.od.nih.gov/pdf/factsheets/ VitaminC-Consumer.pdf (accessed on 4 October 2020).

6. Granger, M.; Eck, P. Dietary Vitamin C in Human Health. Adv. Food Nutr. Res. 2018, 83, 281-310. [PubMed]

7. Gombart, A.F.; Pierre, A.; Maggini, S. A Review of Micronutrients and the Immune System-Working in Harmony to Reduce the Risk of Infection. Nutrients 2020, 12, 236. [CrossRef] [PubMed]

8. Mousavi, S.; Bereswill, S.; Heimesaat, M.M. Immunomodulatory and Antimicrobial Effects of Vitamin C. Eur. J. Microbiol. Immunol. (Bp) 2019, 9, 73-79. [CrossRef]

9. Pullar, J.M.; Carr, A.C.; Vissers, M.C.M. The Roles of Vitamin C in Skin Health. Nutrients. 2017, 9, 866. [CrossRef]

10. Figueroa-Méndez, R.; Rivas-Arancibia, S. Vitamin C in Health and Disease: Its Role in the Metabolism of Cells and Redox State in the Brain. Front. Physiol. 2015, 6, 397. [CrossRef]

11. Aune, D.; Keum, N.; Giovannucci, E.; Fadnes, L.T.; Boffetta, P.; Greenwood, D.C.; Tonstad, S.; Vatten, L.J.; Riboli, E.; Norat, T. Dietary Intake and Blood Concentrations of Antioxidants and the Risk of Cardiovascular Disease, Total Cancer, and All-Cause Mortality: A Systematic Review and Dose-Response Meta-Analysis of Prospective Studies. Am. J. Clin. Nutr. 2018, 108, $1069-1091$. [CrossRef]

12. Woodside, J.V.; Draper, J.; Lloyd, A.; McKinley, M.C. Use of Biomarkers to Assess Fruit and Vegetable Intake. Proc. Nutr. Soc. 2017, 76, 308-315. [CrossRef]

13. Institute of Medicine. Dietary Reference Intakes for Vitamin C, Vitamin E, Selenium, and Caroteinoids; Food and Nutrition Board; National Academy Press: Washington, DC, USA, 2000.

14. Maxfield, L.; Crane, J.S. Vitamin C Deficiency; StatPearls Publishing: Treasure Island, FL, USA, 2020.

15. U.S. Department of Agriculture; U.S. Department of Health and Human Services. Dietary Guidelines for Americans; Government Printing Office: Washington, DC, USA, 2005.

16. United States Department of Agriculture; United States Department of Health and Human Services. Dietary Guidelines for Americans. Available online: https://health.gov/sites/default/files/2020-01/DietaryGuidelines2010.pdf (accessed on 15 October 2020).

17. Centers for Disease Control and Prevention. NHANES Response Rates and Population Totals. Available online: https://wwwn. cdc.gov/nchs/nhanes/responserates.aspx\#response-rates (accessed on 13 December 2020).

18. Centers for Disease Control and Prevention. National Health and Nutrition Examination Survey. Available online: https: //www.cdc.gov/nchs/nhanes/index.htm (accessed on 22 October 2020).

19. Freedman, L.S.; Commins, J.M.; Willett, W.; Tinker, L.F.; Spiegelman, D.; Rhodes, D.; Potischman, N.; Neuhouser, M.L.; Moshfegh, A.J.; Kipnis, V.; et al. Evaluation of the 24-Hour Recall as a Reference Instrument for Calibrating Other Self-Report 
Instruments in Nutritional Cohort Studies: Evidence from the Validation Studies Pooling Project. Am. J. Epidemiol. 2017, 186, 73-82. [CrossRef]

20. Centers for Disease Control and Prevention. National Health and Nutrition Examination Survey (NHANES), MEC in-Person Dietary Interviewers Procedures Manual. Available online: https://wwwn.cdc.gov/nchs/data/nhanes/2017-2018/manuals/20 17_MEC_In-Person_Dietary_Interviewers_Manual.pdf (accessed on 5 October 2020).

21. United States Department of Agriculture. Food and Nutrient Database for Dietary Studies. Available online: https://www. ars.usda.gov / northeast-area/beltsville-md-bhnrc/beltsville-human-nutrition-research-center/food-surveys-research-group / docs/fndds-download-databases / (accessed on 13 December 2020).

22. Centers for Disease Control and Prevention. National Health and Nutrition Examination Survey 2005-2006 Data Documentation, Codebook and Frequencies Vitamin C. Available online: https://wwwn.cdc.gov/nchs/nhanes/2005-2006/VIC_D.htm (accessed on 13 December 2020).

23. Centers for Disease Control and Prevention. National Health and Nutrition Examination Survey 2017-2018 Data Documentation, Codebook, and Frequencies. Available online: https:/ / wwwn.cdc.gov/Nchs/Nhanes/2017-2018/VIC_J.htm (accessed on 13 December 2020).

24. Schleicher, R.L.; Carroll, M.D.; Ford, E.S.; Lacher, D.A. Serum Vitamin C and the Prevalence of Vitamin C Deficiency in the United States: 2003-2004 National Health and Nutrition Examination Survey (NHANES). Am. J. Clin. Nutr. 2009, 90, 1252-1263. [CrossRef] [PubMed]

25. Kim, S. Overview of Cotinine Cutoff Values for Smoking Status Classification. Int. J. Environ. Res. Public Health 2016, 13, 1236. [CrossRef] [PubMed]

26. Drope, J.; Liber, A.C.; Cahn, Z.; Stoklosa, M.; Kennedy, R.; Douglas, C.E.; Henson, R.; Drope, J. Who's Still Smoking? Disparities in Adult Cigarette Smoking Prevalence in the United States. CA Cancer J. Clin. 2018, 68, 106-115. [CrossRef] [PubMed]

27. Hales, C.M.; Fryar, C.D.; Carroll, M.D.; Freedman, D.S.; Aoki, Y.; Ogden, C.L. Prevalence of Obesity and Severe Obesity among Adults: United States, 2017-2018. NCHS Data Brief 2020, 360, 1-8.

28. Institute of Medicine (US) Committee to Review Dietary Reference Intakes for Vitamin D and Calcium. Dietary Reference Intakes for Calcium and Vitamin D; Taylor, C.L., Ross, A.C., Yaktine, A.L., Eds.; National Academies Press: Washington, DC, USA, 2011.

29. Tooze, J.A.; Midthune, D.; Dodd, K.W.; Freedman, L.S.; Krebs-Smith, S.M.; Subar, A.F.; Guenther, P.M.; Carroll, R.J.; Kipnis, V. A New Statistical Method for Estimating the Usual Intake of Episodically Consumed Foods with Application to Their Distribution. J. Am. Diet. Assoc. 2006, 106, 1575-1587. [CrossRef] [PubMed]

30. National Cancer Institute, Division of Cancer Control \& Population. Usual Dietary Intakes: SAS Macros for the NCI Method. Available online: https:/ / epi.grants.cancer.gov/diet/usualintakes/macros.html (accessed on 13 December 2020).

31. Rehm, C.D.; Peñalvo, J.L.; Afshin, A.; Mozaffarian, D. Dietary Intake among Us Adults, 1999-2012. Jama 2016, $315,2542-2553$. [CrossRef] [PubMed]

32. Liu, J.; Rehm, C.D.; Onopa, J.; Mozaffarian, D. Trends in Diet Quality among Youth in the United States, 1999-2016. Jama 2020, 323, 1161-1174. [CrossRef]

33. Miller, G.; Merlo, C.; Demissie, Z.; Sliwa, S.; Park, S. Trends in Beverage Consumption among High School Students-United States, 2007-2015. MMWR Morb. Mortal. Wkly. Rep. 2017, 66, 112-116. [CrossRef]

34. Miles, G.; Siega-Riz, A.M. Trends in Food and Beverage Consumption among Infants and Toddlers: 2005-2012. Pediatrics 2017, 139, e20163290. [CrossRef]

35. Nicklas, T.A.; O'Neil, C.E.; Saab, R.; Fulgoni, V.L., III. Trends in Orange Juice Consumption and Nutrient Adequacy in Children 2003-2016. Lifesci. Global 2020, 9, 100-114. [CrossRef]

36. Mintel. Juice and Juice Drinks: Including Impact of Covid-19-Us_June. 2020. Available online: https://store.mintel.com/ report/juice-and-juice-drinks-incl-impact-of-covid-19-us-june-2020 (accessed on 8 December 2020).

37. Neves, M.F.; Trombin, V.G.; Marques, V.N.; Martinez, L.F. Global Orange Juice Market: A 16-Year Summary and Opportunities for Creating Value. Trop. Plant Pathol. 2020, 45, 166-174. [CrossRef]

38. Smedman, A.; Lindmark-Månsson, H.; Drewnowski, A.; Edman, A.K.M. Nutrient Density of Beverages in Relation to Climate Impact. Food Nutr. Res. 2010, 54, 5170. [CrossRef] [PubMed]

39. Guasch-Ferré, M.; Hu, F.B. Are Fruit Juices Just as Unhealthy as Sugar-Sweetened Beverages? Jama Netw. Open 2019, 3, e193109. [CrossRef] [PubMed]

40. Heyman, M.B.; Abrams, S.A. Fruit Juice in Infants, Children, and Adolescents: Current Recommendations. Pediatrics 2017, 139, e20170967. [CrossRef] [PubMed]

41. Brauchla, M.; Rehm, C.D.; Fulgoni, V.L., III. Cost-Effective Options for Increasing Consumption of Underconsumed Food Groups and Nutrients in the US. Public Health Nutr. 2021, in press.

42. Miller, V.; Yusuf, S.; Chow, C.K.; Dehghan, M.; Corsi, D.J.; Lock, K.; Popkin, B.; Rangarajan, S.; Khatib, R.; Lear, S.A.; et al Availability, Affordability, and Consumption of Fruits and Vegetables in 18 Countries across Income Levels: Findings from the Prospective Urban Rural Epidemiology (Pure) Study. Lancet Glob. Health 2016, 4, e695-e703. [CrossRef]

43. Mook, K.; Laraia, B.A.; Oddo, V.M.; Jones-Smith, J.C. Food Security Status and Barriers to Fruit and Vegetable Consumption in Two Economically Deprived Communities of Oakland, California, 2013-2014. Prev. Chronic Dis. 2016, 13, E21. [CrossRef]

44. Rehm, C.D.; Monsivais, P.; Drewnowski, A. Relation between Diet Cost and Healthy Eating Index 2010 Scores among Adults in the United States 2007-2010. Prev. Med. 2015, 73, 70-75. [CrossRef] 
45. Jack, D.; Neckerman, K.; Schwartz-Soicher, O.; Lovasi, G.S.; Quinn, J.; Richards, C.; Bader, M.; Weiss, C.; Konty, K.; Arno, P.; et al. Socio-Economic Status, Neighbourhood Food Environments and Consumption of Fruits and Vegetables in New York City. Public Health Nutr. 2013, 16, 1197-1205. [CrossRef]

46. Blitstein, J.L.; Snider, J.; Evans, W.D. Perceptions of the Food Shopping Environment Are Associated with Greater Consumption of Fruits and Vegetables. Public Health Nutr. 2012, 15, 1124-1129. [CrossRef]

47. Haynes-Maslow, L.; Parsons, S.E.; Wheeler, S.B.; Leone, L.A. A Qualitative Study of Perceived Barriers to Fruit and Vegetable Consumption among Low-Income Populations, North Carolina, 2011. Prev. Chronic. Dis. 2013, 10, E34. [CrossRef] [PubMed]

48. Lucan, S.C.; Barg, F.K.; Long, J.A. Promoters and Barriers to Fruit, Vegetable, and Fast-Food Consumption among Urban, Low-Income African Americans-A Qualitative Approach. Am. J. Public Health 2010, 100, 631-635. [CrossRef] [PubMed]

49. Lee-Kwan, S.H.; Moore, L.V.; Blanck, H.M.; Harris, D.M.; Galuska, D. Disparities in State-Specific Adult Fruit and Vegetable Consumption-United States, 2015. MMWR Morb. Mortal. Wkly Rep. 2017, 66, 1241-1247. [CrossRef] [PubMed]

50. U.S. Department of Agriculture. Interactive Chart: Most and Least Expensive Fruits and Vegetables. Available online: https: / / www.ers.usda.gov / data-products / fruit-and-vegetable-prices / interactive-chart-most-and-least-expensive-fruits-andvegetables/ (accessed on 13 December 2020).

51. United States Department of Agriculture. The Estimated Amount, Value, and Calories of Postharvest Food Losses at the Retail and Consumer Levels in the United States; Wells, H.F., Buzby, J.C., Hyman, F., Eds.; United States Department of Agriculture: Washington, DC, USA, 2014. Available online: https:/ / www.ers.usda.gov/webdocs/publications/43833/43680_eib121.pdf?v=96 67.8 (accessed on 10 December 2020).

52. American Heart Association. Vitamin Supplements: Hype or Help for Healthy Eating. Available online: https://www.heart.org/ en/healthy-living/healthy-eating/eat-smart/nutrition-basics/vitamin-supplements-hype-or-help-for-healthy-eating (accessed on 8 December 2020).

53. American Institute for Cancer Research. Supplements and Nutrients, Accept No Substitutes. Available online: https://www.aicr. org/cancer-prevention/healthy-eating/supplements-nutrients / (accessed on 13 December 2020).

54. Marra, M.V.; Boyar, A.P. Position of the American Dietetic Association: Nutrient Supplementation. J. Am. Diet. Assoc. 2009, 85, 257S-264S.

55. US Preventive Services Task Force. Vitamin Supplementation to Prevent Cancer and CVD: Preventive Medication. Available online: https://www.uspreventiveservicestaskforce.org/uspstf/document/RecommendationStatementFinal/vitaminsupplementation-to-prevent-cancer-and-cvd-counseling (accessed on 23 November 2020).

56. Bailey, R.L.; Gahche, J.J.; Miller, P.E.; Thomas, P.R.; Dwyer, J.T. Why US Adults Use Dietary Supplements. JAMA Intern. Med. 2013, 173, 355-361. [CrossRef]

57. Kantor, E.D.; Rehm, C.D.; Du, M.; White, E.; Giovannucci, E.L. Trends in Dietary Supplement Use among US Adults from 1999-2012. Jama 2016, 316, 1464-1474. [CrossRef]

58. Stierman, B.; Mishra, S.; Gahche, J.J.; Potischman, N.; Hales, C.M. Dietary Supplement Use in Children and Adolescents Aged $\leq$ 19 Years-United States, 2017-2018. MMWR Morb. Mortal. Wkly. Rep. 2020, 69, 1557-1562. [CrossRef]

59. Lobo, V.; Patil, A.; Phatak, A.; Chandra, N. Free Radicals, Antioxidants and Functional Foods: Impact on Human Health. Pharmacogn. Rev. 2010, 4, 118-126. [CrossRef]

60. Cowan, A.E.; Jun, S.; Tooze, J.A.; Eicher-Miller, H.A.; Dodd, K.W.; Gahche, J.J.; Guenther, P.M.; Dwyer, J.T.; Potischman, N.; Bhadra, A.; et al. Total Usual Micronutrient Intakes Compared to the Dietary Reference Intakes among U.S. Adults by Food Security Status. Nutrients 2019, 12, 38. [CrossRef]

61. Teucher, B.; Olivares, M.; Cori, H. Enhancers of Iron Absorption: Ascorbic Acid and Other Organic Acids. Int. J. Vitam. Nutr. Res. 2004, 74, 403-419. [CrossRef]

62. Aschemann-Witzel, J.; Gantriis, R.F.; Fraga, P.; Perez-Cueto, F.J. Plant-Based Food and Protein Trend from a Business Perspective: Markets, Consumers, and the Challenges and Opportunities in the Future. Crit. Rev. Food Sci. Nutr. 2020, 1-10. [CrossRef] [PubMed]

63. Shan, Z.; Rehm, C.D.; Rogers, G.; Ruan, M.; Wang, D.D.; Hu, F.B.; Mozaffarian, D.; Zhang, F.F.; Bhupathiraju, S.N. Trends in Dietary Carbohydrate, Protein, and Fat Intake and Diet Quality among Us Adults, 1999-2016. JAMA 2019, 322, 1178-1187. [CrossRef] [PubMed]

64. Willett, W. Nutritional Epidemiology, 3rd ed.; New York Oxford University Press: New York, NY, USA, 2012. 